\title{
Multiplication onset and electric field properties of proton-irradiated LGADs
}

\author{
Sofía Otero Ugobono ${ }^{* 1,2}$, M. Centis Vignali ${ }^{1}$, M. Fernández García ${ }^{1,3}$, C. Gallrapp ${ }^{1}$, \\ S. Hidalgo Villena ${ }^{4}$, I. Mateu ${ }^{1}$, M. Moll ${ }^{1}$, G. Pellegrini ${ }^{4}$, A. Ventura Barroso ${ }^{1,5}$, I. Vila ${ }^{3}$ \\ ${ }^{1}$ CERN \\ ${ }^{2}$ Universidade de Santiago de Compostela \\ ${ }^{3}$ Universidad de Cantabria \\ ${ }^{4}$ Centre Nacional de Microelectrònica (IMB-CNM-CSIC) \\ ${ }^{5}$ Universitat de Barcelona
}

E-mail: sofia.otero.ugobonodcern.ch

\begin{abstract}
This work focuses on the study of a set of Low Gain Avalanche Detectors (LGADs) produced by CNM, Barcelona (run 7859). Several samples were irradiated with 24-GeV/c protons up to different fluences, ranging between $10^{12}$ and $10^{15} \mathrm{n} e q / \mathrm{cm}^{2}$. This study concentrates mainly on the LGADs irradiated up to $10^{14} \mathrm{n}_{\mathrm{eq}} / \mathrm{cm}^{2}$. The measurements performed to characterise the devices include TCT, edge-TCT, TPA-TCT, and CV/IV measurements. The main goals of these studies were to analyse the voltage required to fully deplete the multiplication layer of LGADs; to measure gain degradation; and to investigate the distribution of the electric field inside the devices after irradiation, as well as the characteristics of the space charge. In order to do so, the measurements were performed under different temperature, read-out and biasing conditions. The obtained data confirm that in highly proton-irradiated LGADs the depletion of the bulk starts from the back electrode, thus shifting the onset of charge multiplication towards higher voltages. This is caused by a space charge sign inversion that is in turn accompanied by the appearance of a triple junction. Furthermore, it was found that annealing causes a recovery of gain along with a reduction of the multiplication-onset voltage towards unirradiated-like values.
\end{abstract}

The 26th International Workshop on Vertex Detectors

10-15 September, 2017

Las Caldas, Asturias, Spain

${ }^{*}$ Speaker. 


\section{Introduction}

The High Luminosity upgrade of the CERN Large Hadron Collider (HL-LHC) presents several technological challenges. In particular the increase in luminosity causes a rise in the expected pile-up. To cope with it silicon sensors with better timing capabilities ( $\sim 10 \mathrm{ps})$ are being developed [1]. One of the technologies under consideration is that of Low Gain Avalanche Detectors (LGADs) [2]. Said devices are characterised by their intrinsic charge gain. The internal multiplication of LGADs should improve their timing capabilities as well as increase their signal height, even after irradiation. The latter is an important point given that the sensors must be radiation tolerant, specifically they must withstand fluences of up to $10^{15} \mathrm{n}_{\mathrm{eq}} / \mathrm{cm}^{2}$. Several production runs of LGADs have already been fabricated by CNM-Barcelona. Moreover, during the past years there has been a continuous effort to conduct radiation-hardness and timing tests on thick $(\sim 300 \mu \mathrm{m})$ and thin ( $\sim 50 \mu \mathrm{m}$ ) LGADs $[3,4,5,6,7,8]$. The development of LGADs is considerably advanced, with ATLAS and CMS already foreseeing the use of LGADs in their detectors [9, 10, 11]. The main goal of this study is, thus, to better understand the effects of proton irradiation on LGADs, particularly the change in space charge and electric-field behaviour.

\section{LGAD structure and sample description}

Low Gain Avalanche Detectors are planar silicon sensors that consist of a p-type bulk with a p++ ohmic contact, an $\mathrm{n}++$ electrode, and a diffused $\mathrm{p}+$ (multiplication) layer just below the $\mathrm{n}++$ electrode, see Fig. 1a. The presence of this $\mathrm{p}+$ layer implies that, when reverse biased, a high electric field is created there. Whenever charge carriers pass through that region they will undergo impact ionisation, thus producing additional charge carriers. This process can only occur if the electric field is high enough $(>200 \mathrm{kV} / \mathrm{cm})$. As a result, the gain of the device is directly dependent on the bias voltage, the device temperature and the doping concentration of the p+layer [12].

The set of analysed samples consists of pad PiN diodes (no multiplication layer) and LGADs from run 7859, manufactured by CNM in Barcelona. All devices coming from a run are produced with the same mask design, yet the multiplication layer doping can change amongst wafers. These PiN diodes and LGADs were produced on p-doped float-zone silicon wafers with a resistivity of $12 \mathrm{k} \Omega \cdot \mathrm{cm}$. Each sensor has an active area of $3 \times 3 \mathrm{~mm}^{2}$, a thickness of $285 \mu \mathrm{m}$, a guard ring (GR), and a junction termination extension (JTE) (see Fig. 1a). The JTE is a deep n-type diffusion that overlaps with the edge of the main junction, between the $\mathrm{n}++$ electrode and the multiplication layer. Its purpose is to increase the operational voltage range by making the electric field more homogeneous at the junction [13]. In order to allow for laser measurements, each sample has openings in the metallisation, both on the front and the back side.

The samples studied come from four wafers (W). W1 and W2 have the same multiplicationlayer implant dose: $1.8 \times 10^{13} \mathrm{~cm}^{-2}$. Correspondingly, W3 and W4 have both a multiplicationlayer implant dose of $2.0 \times 10^{13} \mathrm{~cm}^{-2}$. Regarding electrical parameters, all sensors have an end capacitance before irradiation of $\sim 4.1 \mathrm{pF}$ and a total leakage current (pad + GR currents) of less than $0.3 \mu \mathrm{A}$ at full depletion voltage $\left(V_{\mathrm{dep}}\right)$ and $20^{\circ} \mathrm{C}$.

The irradiation campaign with $24-\mathrm{GeV} / \mathrm{c}$ protons took place at the CERN IRRAD facility (hardness factor $\sim 0.56$, in 2015) [14],[15]. The detectors were separated in four sets of four 


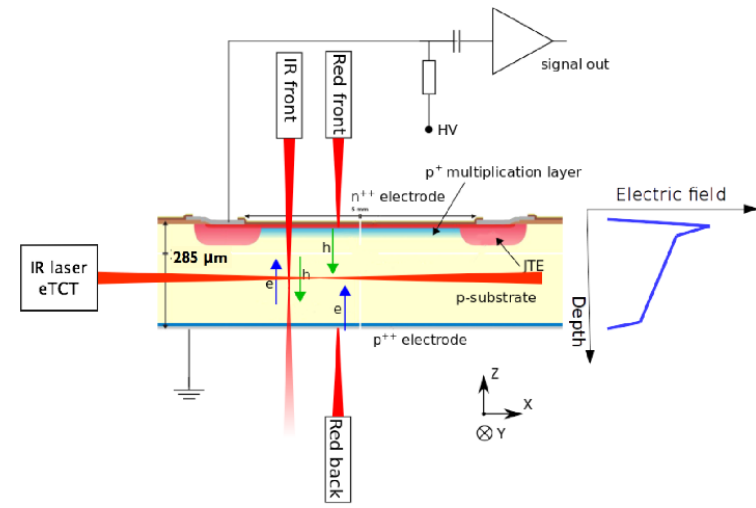

(a)

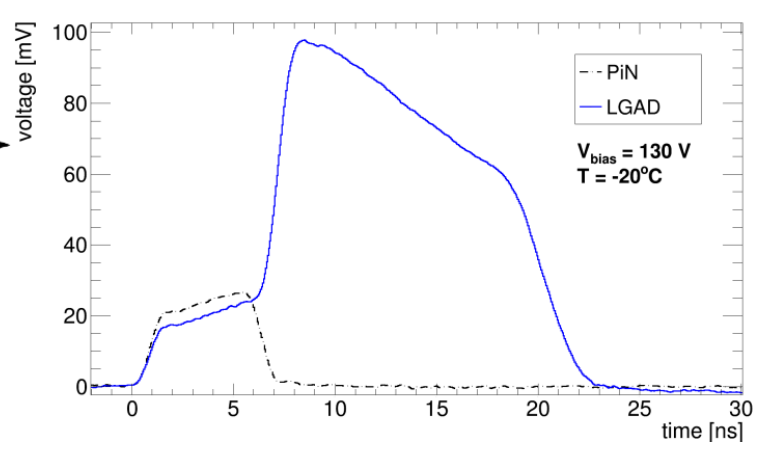

(b)

Figure 1: (a) Cross-section schematic of an LGAD. The laser configuration of the TCT and eTCT set-up can also be seen. On the right, a depiction of the electric field inside an LGAD is shown. (b) Waveforms obtained with red back TCT on an LGAD (solid line) and on a PiN diode (dashed line).

detectors each. Every set was composed of 2 PiN diodes and 2 LGADs, each pair formed by one sample from $\mathrm{W} 1$ or $\mathrm{W} 2$ and another one from $\mathrm{W} 3$ or W4. Each set was irradiated up to a different fluence: $10^{12}, 10^{13}, 10^{14}$, and $10^{15} \mathrm{n}_{\mathrm{eq}} / \mathrm{cm}^{2}$. After irradiation, and before their characterisation, the detectors were annealed at $60^{\circ} \mathrm{C}$ for $80 \mathrm{~min}$. Most of the studies here presented focus on the LGADs irradiated up to a fluence of $10^{14} \mathrm{n}_{\mathrm{eq}} / \mathrm{cm}^{2}$.

\section{Experimental techniques}

To characterise the devices, four methods were employed: IV measurements, i.e. leakage current as a function of voltage; Transient Current Technique (TCT) [16]; edge-TCT [17]; and Two Photon Absorption (TPA) TCT [18]. The methods as implemented in the present work follow.

\subsection{Transient Current Technique}

TCT consists in the generation of charge carriers by illuminating silicon sensors with 200-ps-wide laser pulses (see Fig. 1a). For this particular study, red $(660 \mathrm{~nm})$ laser pulses were used. Since the absorption length of red light in silicon is of a few micrometers, the red laser injects electrons (back-side, p++ electrode, illumination) or holes (front-side, $\mathrm{n}++$ electrode, illumination) close to the illuminated surface. This property makes red light TCT a useful tool to characterise the electric field, and the movement of electrons $\left(\mathrm{e}^{-}\right)$and holes $\left(\mathrm{h}^{+}\right)$inside a device. Although not included in this study, it is possible to perform infrared (IR) TCT with front-side or back-side illumination. Due to the absorption length of IR light in silicon $(\sim 1 \mathrm{~mm})$ this type of TCT is used to mimic the behaviour of the device under test (DUT) when exposed to minimum ionising particles (MIPs).

When performing red back TCT ( $\mathrm{e}^{-}$injection) on an n-in-p diode (PiN) or an LGAD, the signals obtained are significantly different. Fig. $1 \mathrm{~b}$ shows the signal obtained, at $-20^{\circ} \mathrm{C}$ and $130 \mathrm{~V}$, with a PiN diode $\left(V_{\text {dep }} \sim 44 \mathrm{~V}\right)$ and an LGAD $\left(V_{\text {dep }} \sim 71 \mathrm{~V}\right)$ from run 7859 , W3 and W4 respectively (both wafers have the same properties). The LGAD signal is composed of an initial low-amplitude pulse due to the drift of the injected electrons, which coincides with the full signal of the PiN. In the case of the LGAD signal, right after the electron-drift induced pulse there is the beginning of the 
multiplication. Said multiplication ends at about $8 \mathrm{~ns}$, only to be followed by the drift of the holes produced during multiplication (known as secondary holes). The tail of the signal pulse, starting at $\sim 19 \mathrm{~ns}$, is caused by the read-out electronics.

\subsection{Edge-TCT}

This technique is also based on the light-induced generation of charge carriers, albeit by means of 200-ps infrared laser pulses and edge illumination (in contrast with the front or back illumination explained in the previous section). The side illumination characteristic of eTCT and the penetration length of IR light in silicon result in eTCT being a method for probing the electric field all through the thickness of the device (see Fig. 1a).

\subsection{Two Photon Absorption TCT}

The theoretical basis for Two Photon Absorption (TPA) TCT is that due to the time-energy uncertainty principle it is possible to produce an $\mathrm{e}^{-} / \mathrm{h}^{+}$pair by means of the absorption of two photons, each with energy below that of the bandgap. Two photons that individually possess an energy lower than that of the bandgap, but that the sum of both their energies is equal or higher to it, could be absorbed by means of an intermediate virtual state. For this to be possible, both photons must interact with the $\mathrm{e}^{-}$in an interval of less than $1 \mathrm{fs}$ [19]. Experimentally this is achieved by means of a femtosecond pulsed mode-locked laser. Its focal point is an ellipsoid with a section of $\sim 1 \mu \mathrm{m}$, and a length of $\sim 10 \mu \mathrm{m}$. Since the condition $\Delta t<1 \mathrm{fs}$ is only satisfied in the focal point of the laser, the electron-hole generation is extremely localised, this allows a three-dimensional probing of the electric field [18].

\section{Characterisation}

The characterisation of LGADs and PiN diodes from run 7859 was done by performing IV measurements, TCT voltage scans, eTCT and TPA-TCT Z-scans at different voltages. The study is particularly focused on the devices irradiated up to a fluence of $10^{14} \mathrm{n}_{\mathrm{eq}} / \mathrm{cm}^{2}$. The objective is to analyse the effects of radiation on several properties of LGADs, specifically the gain; the multiplication onset; and the evolution of the electric field, the collected charge, and the space charge. The main use of including PiN diodes in this study is to serve as a reference for gain calculations.

\subsection{Charge collection with voltage}

In this section the results obtained from red TCT voltage scans on all devices, before and after irradiation, are presented. The procedure followed during a voltage scan is simple. The position of the laser beam spot remains constant whilst the bias voltage applied to the DUT is changed. Voltage scans are a means of determining the variation of charge collection with voltage, and of obtaining information regarding the evolution of the electric field with voltage. Additionally, this type of measurement can be used to calculate the gain of an LGAD. This is done by comparing the collected charge of an LGAD to that of a PiN diode at a given temperature and voltage. For future reference, it should be clarified that the GR was left floating during these measurements, this is the usual procedure for TCT measurements. 


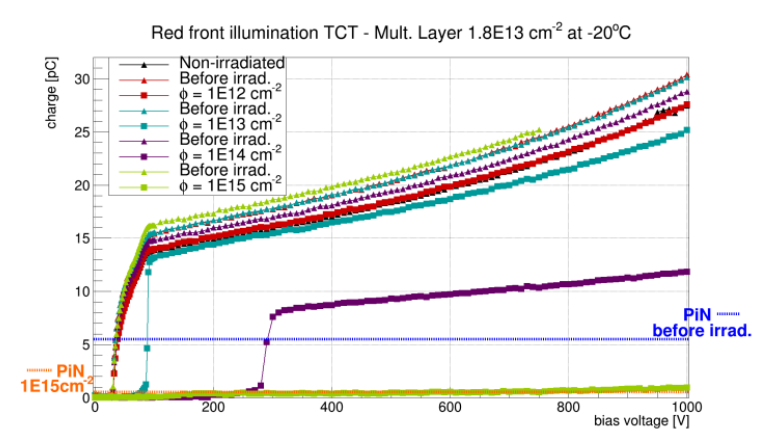

(a)

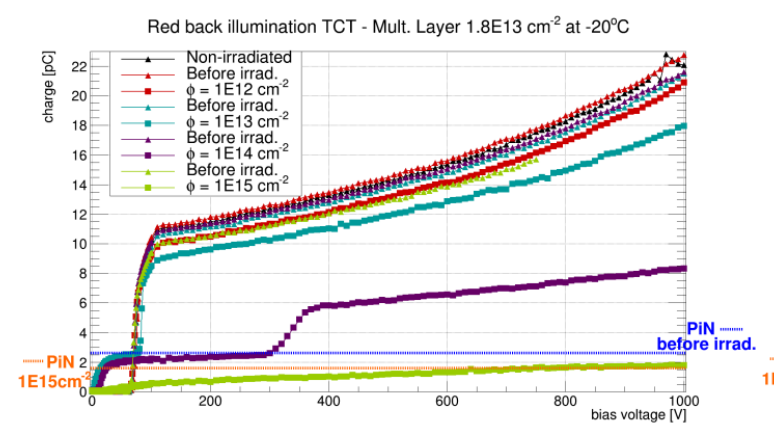

(c)

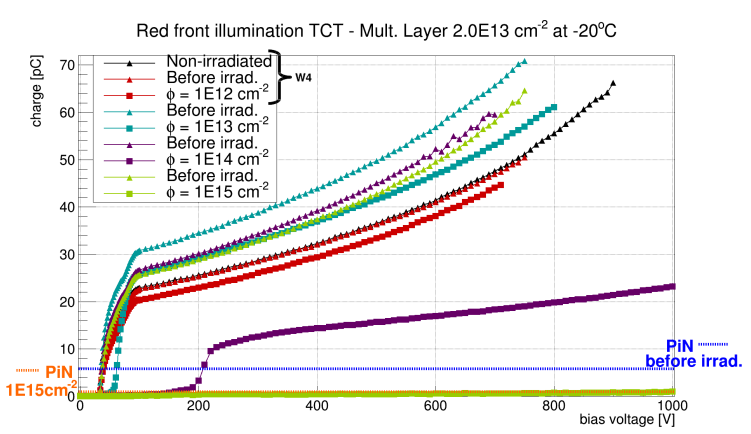

(b)

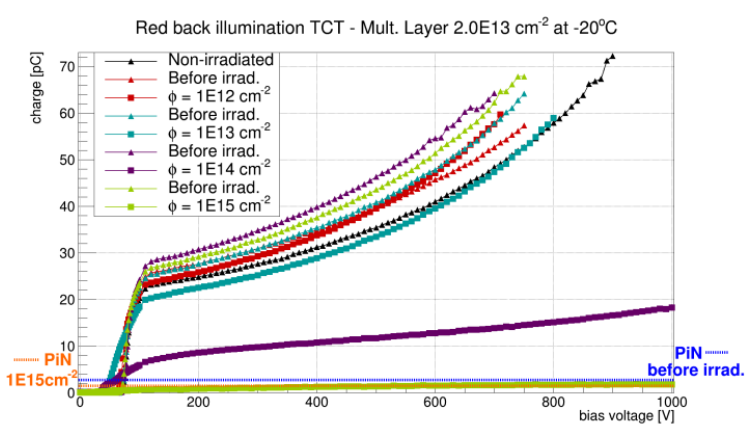

(d)

Figure 2: Charge collection in LGADs as a function of voltage at $-20^{\circ} \mathrm{C}$ both before and after proton irradiation. Results for LGADs from W1 and W2 (multiplication layer dose: $1.8 \times 10^{13} \mathrm{~cm}^{-2}$ ) with hole injection (a), and electron injection (c). Results for LGADs from W3 and W4 (multiplication layer dose: $2.0 \times 10^{13} \mathrm{~cm}^{-2}$ ) with hole injection (b), and electron injection (d). The dashed lines indicate the maximum charge collection observed with the reference PiN diodes before irradiation (blue) and after proton irradiation to $10^{15} \mathrm{n}_{\mathrm{eq}} / \mathrm{cm}^{2}$ (orange).

The obtained plots for charge collection in LGADs as a function of voltage at $-20^{\circ} \mathrm{C}$ can be seen in Fig. 2. Also included in Fig. 2 are the collected-charge values for a PiN diode irradiated up to $10^{15} \mathrm{n}_{\mathrm{eq}} / \mathrm{cm}^{2}$, and for a non-irradiated one. The considerable difference in charge collection due to internal multiplication between LGADs and PiN diodes can be clearly seen. However, a decrease in charge collection with fluence is observed for LGADs and PiN diodes. In fact, at a fluence of $10^{15} \mathrm{n}_{\mathrm{eq}} / \mathrm{cm}^{2}$ the charge collected by an LGAD is roughly the same as that of an equally irradiated PiN. It should be noted that in the case of the PiN diodes, the charge collected is different for each illumination (red front or red back) due to a difference in intensity between branches of the optical system ${ }^{1}$.

Another visible feature in the voltage scans for hole injection (Fig. 2a and Fig. 2b) is that at low voltages the charge collection is nearly null and then it increases dramatically. Before irradiation the region of almost no charge collection at the beginning of the curve stretches over a span of $30 \mathrm{~V}$ for $\mathrm{W} 1 / \mathrm{W} 2$, and $32 \mathrm{~V}$ for W3/W4. After this initial foot, the charge collection escalates. The

\footnotetext{
${ }^{1}$ Because of this and of possible intensity fluctuations between measurements, when performing gain calculations the charge collection must be normalised by the laser intensity. By means of a reference photodiode, the laser intensity is continuously monitored during TCT measurements. These data are stored together with that from the DUT.
} 


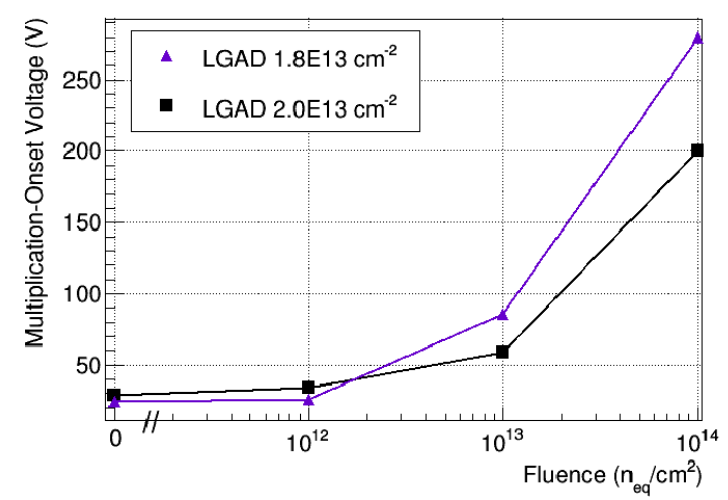

Figure 3: Multiplication-onset voltage as a function of fluence at $-20^{\circ} \mathrm{C}$.

explanation for this threshold voltage ensues from the internal structure of LGADs. The voltage at which the charge collection begins to increase in hole injection (threshold voltage) is the voltage at which impact ionisation begins, i.e. the multiplication-onset voltage. Ergo, the threshold voltage coincides with the bias voltage required to fully deplete the $\mathrm{p}+$ (multiplication) layer. In a previous study, similar LGADs were irradiated at several fluences with neutrons, $200 \mathrm{MeV} / \mathrm{c}$ pions, and $800 \mathrm{MeV} / \mathrm{c}$ protons [3]. In said study it was found that the multiplication-onset voltage decreases with fluence. This effect would be a consequence of what is generally referred to as acceptor removal, i.e. the decrease in effective acceptor concentration $\left(N_{a}\right)$. A decrease in the effective doping of the multiplication layer implies a decrease of the voltage required to deplete it $\left(V_{\mathrm{dep}} \propto N_{a}\right.$ [20]).

Contrary to what was observed in [3], in our study, where LGADs were irradiated with more energetic protons $(24 \mathrm{GeV} / \mathrm{c})$, it was found that the threshold voltage increases with fluence, see Fig. 3. A possible explanation is a change in space charge due to additional space charge introduced by radiation-induced defects. Before irradiation the depletion region grows from the front side of the device, where the junction between the $\mathrm{n}++$ implant and the $\mathrm{p}+$ multiplication layer is. As a result of irradiation, deep-level traps appear in the silicon bulk. Said traps can capture electrons and holes. If enough holes get trapped or defects are created that introduce positive space charge, then the space charge sign will change [21]. Such an inversion of space charge sign would cause the formation of a pn-junction on the back of the sensor. The depletion of the sensor would then begin from the back junction rather than from the frontal pn-junction at the p+layer region. As a consequence the signal multiplication can only be observed when both the bulk and the multiplication layer are fully depleted. Such an effect would explain the increase of the multiplication-onset voltage with fluence.

Further confirmation of the presence of a back junction in these devices is found in the voltage scans obtained through electron injection, i.e. red back illumination TCT (Fig. 2c and Fig. 2d). If the depletion region grows from the front side, as before irradiation, then at low voltages no charge collection will be observed with red back illumination. Since red light has an absorption length in silicon of a few $\mu \mathrm{m}$, before full depletion the $\mathrm{e}^{-} / \mathrm{h}^{+}$pairs produced recombine and induce no signal. It is not until the device is fully depleted that the $\mathrm{e}^{-} / \mathrm{h}^{+}$pairs can drift and induce a signal. On the other hand, if after irradiation there is a change in space charge that causes the depletion region to grow from the back side of the sensor, then charge collection will begin at low voltages. 


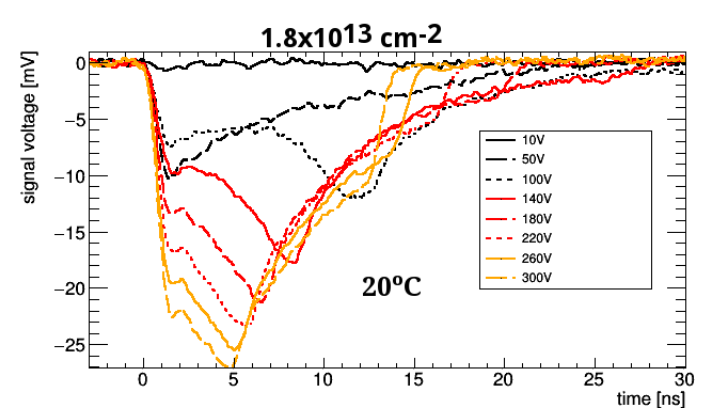

(a)

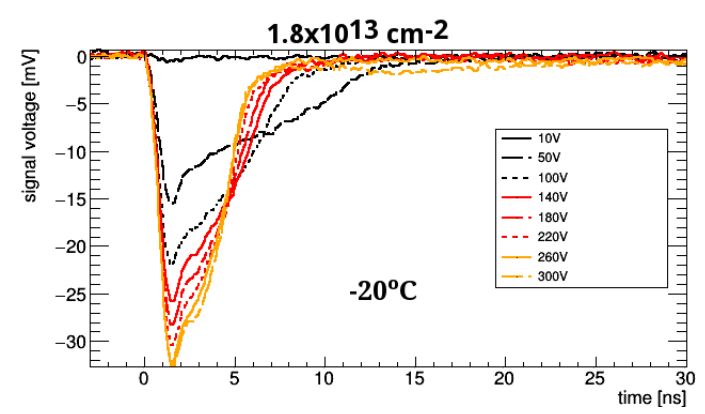

(c)

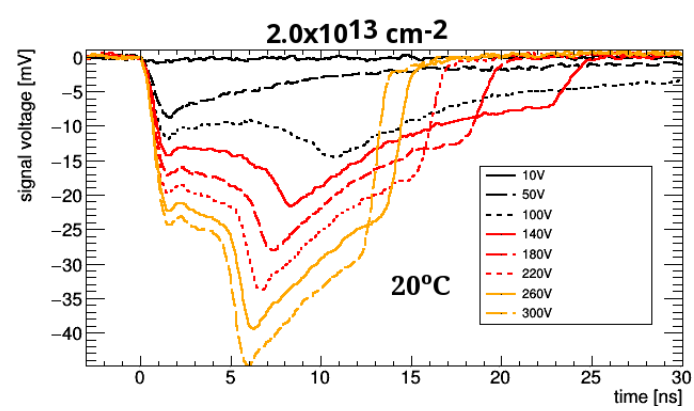

(b)

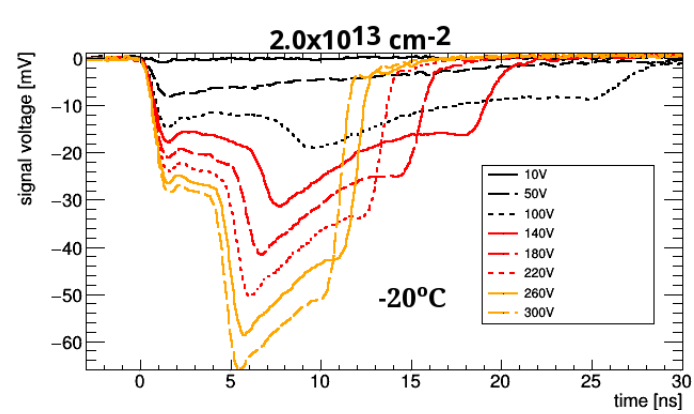

(d)

Figure 4: Waveforms obtained through $\mathrm{e}^{-}$injection at $20^{\circ} \mathrm{C}$ (top) and $-20^{\circ} \mathrm{C}$ (bottom) with the LGADs from W2 (left), and W3 (right) irradiated up to $10^{14} \mathrm{n}_{\mathrm{eq}} / \mathrm{cm}^{2}$. W2 has a multiplication layer dose of $1.8 \times 10^{13} \mathrm{~cm}^{-2}$. W3 has a multiplication layer dose of $2.0 \times 10^{13} \mathrm{~cm}^{-2}$.

In Fig. $2 \mathrm{c}$ and Fig. $2 \mathrm{~d}$ it can be seen that at a fluence of $10^{13} \mathrm{n}_{\mathrm{eq}} / \mathrm{cm}^{2}$ or above, charge collection starts at low voltages.

\subsection{Waveforms at different temperatures}

In order to have more conclusive proof of the mentioned inversion of space charge sign, a study of the waveforms obtained in red TCT voltage scans at different temperatures was carried out. The motivation for this is that the occupation probability of traps is highly dependent on temperature. The emission probability of holes from deep-level traps decreases with decreasing temperature, ergo the lower the temperature the longer the holes remain trapped [20]. Consequently, if the inversion of space charge sign is due to hole trapping, the effect should be reduced at higher temperatures. This means that if after irradiation the depletion region grows from the back at $-20^{\circ} \mathrm{C}$, as seen in section 4.1 , at $20^{\circ} \mathrm{C}$ the devices might deplete from the front side. Fig. 4 shows the waveforms obtained at $20^{\circ} \mathrm{C}$ and $-20^{\circ} \mathrm{C}$ with the two LGADs irradiated up to $10^{14} \mathrm{n}$ eq $/ \mathrm{cm}^{2}$. The waveforms obtained at low voltages $(\leq 50 \mathrm{~V})$, for both samples and temperatures, manifest the presence of a maximum of the electric field in the back side of the devices, consistent with a back junction. However, and in accordance with the hypothesis of space charge sign inversion due to hole trapping, the effect is most persistent at $-20^{\circ} \mathrm{C}$, particularly in the sample with a lower multiplication layer dose (see Fig. 4c). 


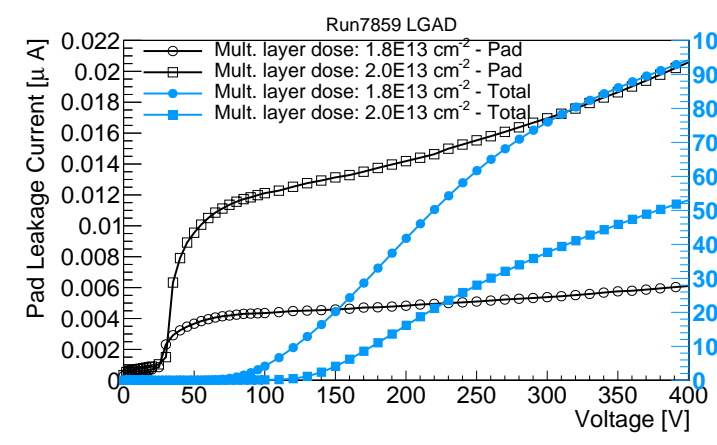

(a)

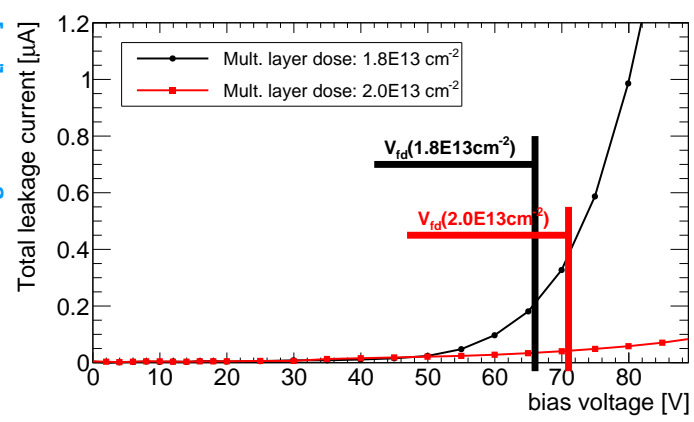

(b)

Figure 5: (a) Pad and total (pad + GR) leakage currents at $20^{\circ} \mathrm{C}$, before irradiation. (b) Detail of the total leakage current around full depletion.

\subsection{Leakage current}

The space charge sign inversion of p-type silicon in LGADs was observed before in [22], however, it was indicated that it only occurred in LGADs with a total leakage current higher than $1 \mu \mathrm{A}$ at full depletion voltage, and $20^{\circ} \mathrm{C}$, before irradiation. According to [22], LGADs with a lower leakage current did not show an inversion of space charge, but a reduction of gain due to acceptor removal in the multiplication layer, as seen in [3]. To verify that the LGADs considered in the present study had a sufficiently low leakage current, where space charge sign inversion is not expected, the before-irradiation IV curves of both devices irradiated up to $10^{14} \mathrm{n}_{\mathrm{eq}} / \mathrm{cm}^{2}$ were carefully inspected. Fig. 5 shows the leakage current as a function of voltage before irradiation at $20^{\circ} \mathrm{C}$ for both LGADs that were later on irradiated to $10^{14} \mathrm{n}_{\mathrm{eq}} / \mathrm{cm}^{2}$. It can be seen, particularly in $5 \mathrm{~b}$, that both devices have a total ( $\mathrm{pad}+\mathrm{GR}$ ) leakage current lower than $1 \mu \mathrm{A}$ at full depletion voltage, and $20^{\circ} \mathrm{C}$.

\subsection{Influence of the guard ring and annealing on TCT scans}

Since TCT measurements until this moment had always been performed without grounding the GR, a thorough study of the possible influence of the GR grounding was performed. Firstly, an unirradiated LGAD kept for reference was tested at $-20^{\circ} \mathrm{C}$ and $20^{\circ} \mathrm{C}$, with and without the GR grounded, see Fig. 6. No GR-induced changes were found. Before irradiation the onset of multiplication voltage remains constant regardless of the temperature (as expected) and connection of the GR.

To properly evaluate the effects of the GR after irradiation various variables were considered, namely: temperature, connection of the GR, multiplication-onset voltage, gain, and date (month and year) when each measurement was performed. In the case of the LGAD from W2 (multiplication layer dose: $1.8 \times 10^{13} \mathrm{~cm}^{-2}$ ), Fig. 7a presents the multiplication-onset voltage measured with and without the GR at $-20^{\circ} \mathrm{C}$, as well as one measurement at $20^{\circ} \mathrm{C}$. In this plot the results are shown with respect to the date when each measurement occurred. When comparing measurements from the same date it is found that at $-20^{\circ} \mathrm{C}$ the GR connection shifts the onset of multiplication. Also, the multiplication-onset voltage is lower at high temperatures, reaching almost the beforeirradiation value of $\sim 30 \mathrm{~V}$. What is most striking in this plot is the decrease in multiplication-onset 


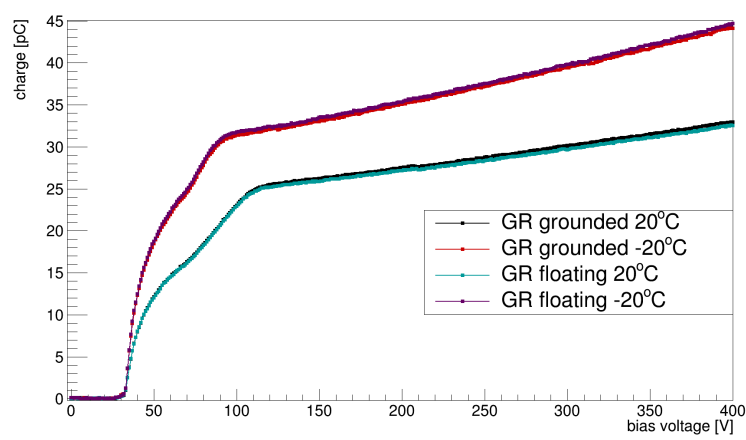

Figure 6: Charge collection as a function of voltage for a non-irradiated LGAD at $20^{\circ} \mathrm{C}$ and $-20^{\circ} \mathrm{C}$, with and without a GR connection.

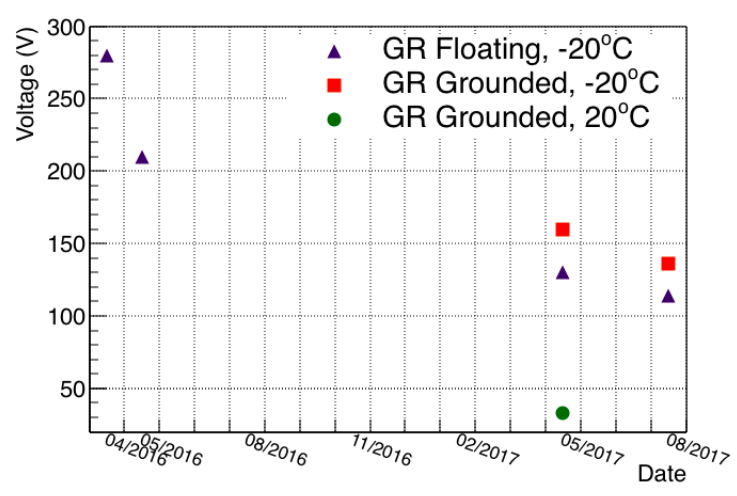

(a)

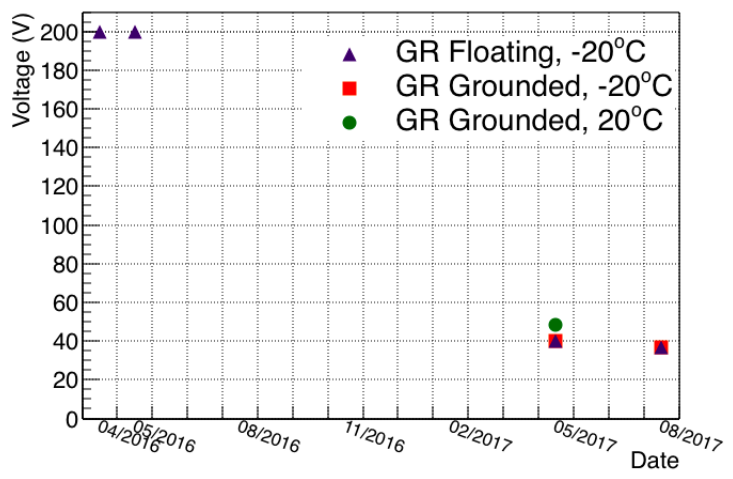

(c)

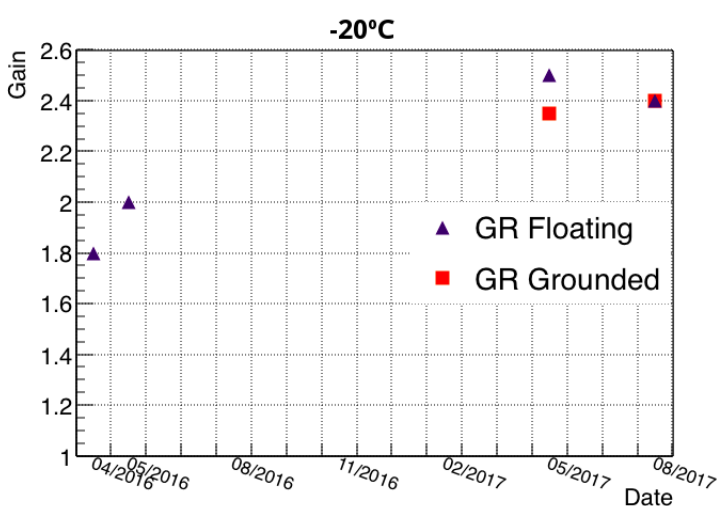

(b)

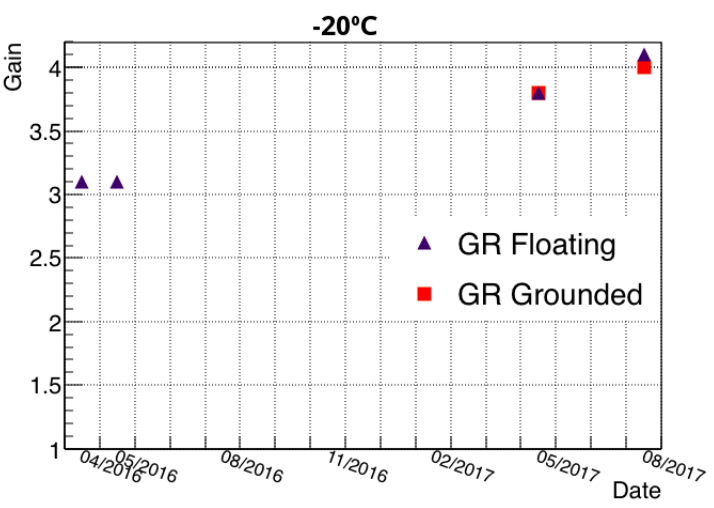

(d)

Figure 7: Evolution with time of the multiplication-onset voltage (left) and the gain at $-20^{\circ} \mathrm{C}$ (right) for the LGADs from W2 (top), and W3 (bottom) irradiated up to $10^{14} \mathrm{neq} / \mathrm{cm}^{2}$.

voltage with time. This change might be related to reverse annealing introducing negative space charge, since in between each set of measurements there were periods of involuntary annealing (due to the transport of the samples, measurements at temperatures above $0^{\circ} \mathrm{C}$, etc.). As regards the influence of the GR on the gain, and its evolution with time, Fig. 7b shows the gain measured at $-20^{\circ} \mathrm{C}$, with and without the GR, as a function of the date when each measurement took place. The 
gain was calculated at $400 \mathrm{~V}$ by comparing the charge collection, normalised by the laser power, of the LGAD with that of a PiN diode from the same wafer and irradiated to the same fluence. Whilst the GR connection scheme does not seem to have much of an effect, the gain increases with time. This would mean that annealing aids the recovery of gain after irradiation. As a matter of fact, the latest measurement presents a gain equal to $70 \%$ of the before-irradiation gain value of $\sim 3.45$ under the same temperature and voltage conditions.

Similarly, the LGAD from W3 (multiplication layer dose: $2.0 \times 10^{13} \mathrm{~cm}^{-2}$ ), presents a multiplication-onset voltage that decreases with time, i.e. with annealing (see Fig. 7c). As a matter of fact, the latest measurements indicate that the multiplication-onset voltage has shifted to values close to that before irradiation, around $32 \mathrm{~V}$. Interestingly, in this sample the GR connection causes no significant changes, and neither does the temperature. Such a behaviour is almost identical to the one expected from a non-irradiated LGAD (Fig. 6). In addition, the gain is also not affected by the GR connection, and with time/annealing it has gone up to about $60 \%$ of the before-irradiation value of $\sim 6.83$.

\subsection{Characterisation of the electric field}

In order to understand how the electric field evolves with voltage and temperature TPA-TCT and eTCT measurements were performed. The LGAD from W3 underwent, in September 2017, eTCT Z-scans, i.e. scanning the device all through its thickness, at different voltages and at $-20^{\circ} \mathrm{C}$ and $20^{\circ} \mathrm{C}$, see Fig. 8 . The results show that temperature does not seem to affect dramatically the profile of the electric field. This is consistent with the behaviour of a non-irradiated LGAD, just as the multiplication onset results commented in section 4.4. However, at $20^{\circ} \mathrm{C}$ there is an unexpected narrow peak in the electric field on the back of the device. Future TPA-TCT scans on the device might shed some light on this peculiarity.

In the case of the LGAD from W2 it was possible, in July 2017, to perform TPA-TCT Z-scans on it, where by front illumination the laser beam spot moves all through the thickness of the sensor. Said scans were performed at $-20^{\circ} \mathrm{C}$ and $0^{\circ} \mathrm{C}$, with bias voltages ranging between 0 and $700 \mathrm{~V}$, see Fig. 9. At both temperatures, $-20^{\circ} \mathrm{C}$ and $0^{\circ} \mathrm{C}$, the electric field clearly begins growing from the back of the device. At $0^{\circ} \mathrm{C}$, already at low voltages $(\sim 30 \mathrm{~V})$ there is also a peak on the front side. In fact, at $\sim 45 \mathrm{~V}$ the electric field on the front side has approximately the same intensity as on the back, giving a profile consistent with a double junction. At $-20^{\circ} \mathrm{C}$, the double peak profile of the electric field appears at $\sim 96 \mathrm{~V}$. Successively, at a voltage between 150 and $200 \mathrm{~V}$ (for both temperatures) the electric field in the front side overpowers the field in the back, resulting in a unique peak on the front. Finally, the electric field at high voltages recovers its intended shape. Space charge sign inversion of p-type silicon due to hole trapping explains the growth of the electric field from the back of the device, as well as the presence of a double junction. Also, it is consistent with the observation that at lower temperatures a higher voltage is needed to increase the intensity of the electric field on the front side of the sensor. However, space charge sign inversion alone does not account for the electric field at higher voltages, nor the intermediate state with just one peak on the front. A comprehensive explanation has not yet been found due to the complexity of the various phenomena at play. 


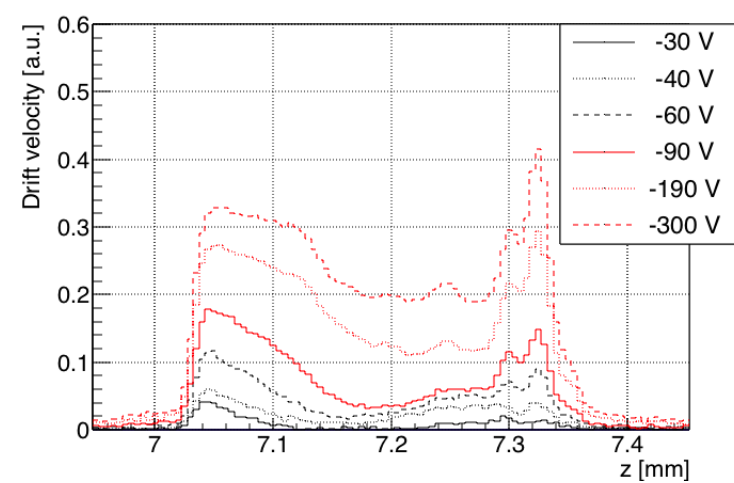

(a)

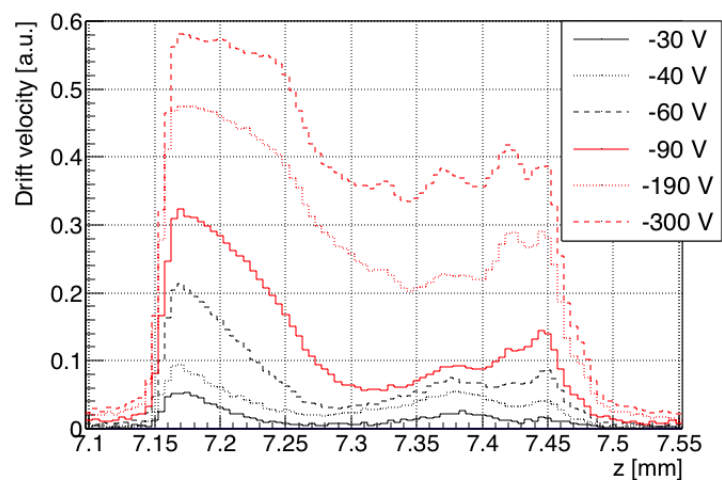

(b)

Figure 8: Drift velocity profiles obtained through eTCT on the LGAD from W3 (mult. layer dose: $2.0 \times$ $10^{13} \mathrm{~cm}^{-2}$ ), irradiated up to $10^{14} \mathrm{n}_{\mathrm{eq}} / \mathrm{cm}^{2}$, at (a) $20^{\circ} \mathrm{C}$ and (b) $-20^{\circ} \mathrm{C}$. In each plot the front side of the device corresponds to the left side of the graph.

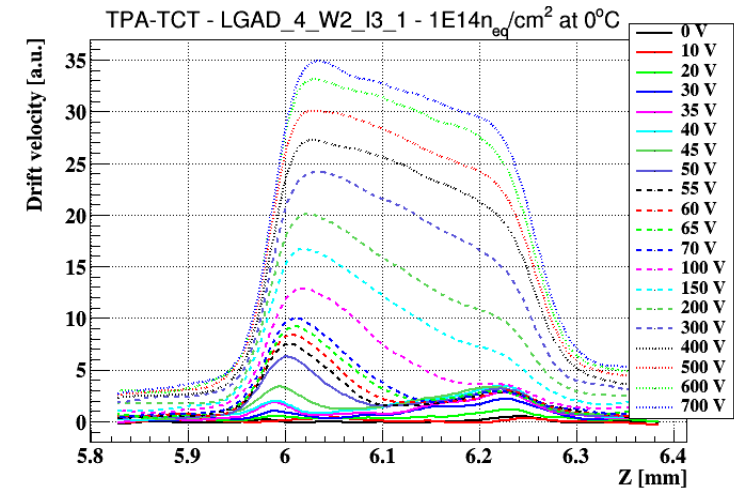

(a)

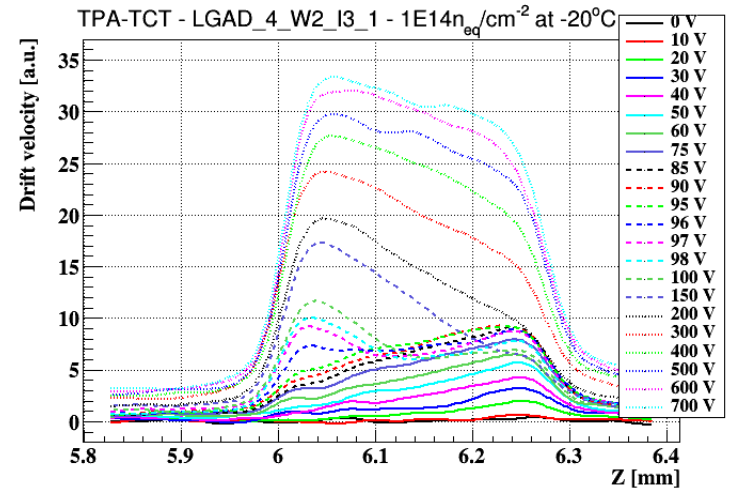

(b)

Figure 9: Drift velocity profiles obtained through TPA-TCT on the LGAD from W2 (mult. layer dose: $1.8 \times 10^{13} \mathrm{~cm}^{-2}$ ), irradiated up to $10^{14} \mathrm{n}_{\mathrm{eq}} / \mathrm{cm}^{2}$, at (a) $0^{\circ} \mathrm{C}$ and (b) $-20^{\circ} \mathrm{C}$. In each plot the front side of the device corresponds to the left side of the graph.

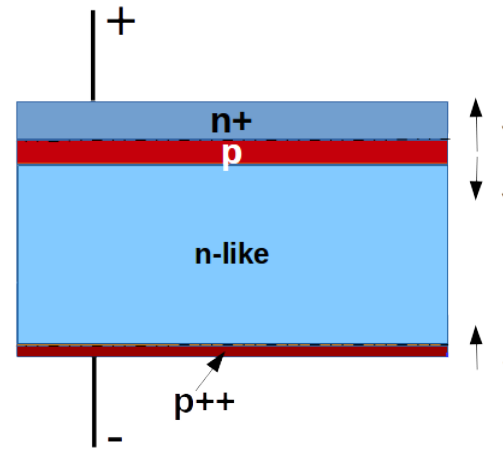

(a)

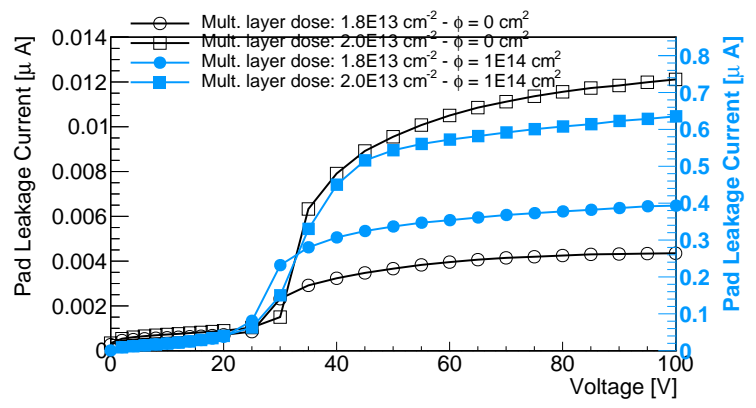

(b)

Figure 10: (a) Schematic of the hypothetical triple-junction structure in an LGAD. (b) Detail at low voltages of the pad leakage current, before and after irradiation, for the two LGADs that were irradiate up to $10^{14} \mathrm{neq} / \mathrm{cm}^{2}$. 
A possible explanation to the observed behaviour could be the presence of a triple junction. Space charge sign inversion of the p-type bulk, resulting in an n-like bulk, in the LGAD could be creating an $n++/ p+/ n / p++$ structure, see Fig. 10a. This would cause the formation of three junctions. One in the front $(\mathrm{n}++/ \mathrm{p}+)$, between the $\mathrm{n}++$ implantation layer of the LGAD and the $\mathrm{p}+$ multiplication layer, which would be reverse biased. One in the back $(n / p++)$, between the $n$-like bulk and the p++ back implantation of the LGAD, which would also be reverse biased. And, finally, one in the middle $(\mathrm{p}+/ \mathrm{n})$, between the $\mathrm{p}+$ multiplication layer and the $\mathrm{n}$-like bulk, which would be forward biased. A potential explanation to the behaviour of the electric field was obtained through a qualitative TCAD simulation of the device in question [23]. According to it, the multiplication layer is depleted at $\sim 30 \mathrm{~V}$, even after irradiation. In spite of this, as higher voltages are applied, the electric field begins growing from the back of the sensor towards the front. It is not until the depletion region reaches the front side that the electric field starts increasing on the front side of the detector, and the standard double-junction effect appears. Afterwards, the space charge starts to change sign from positive to the original negative in the bulk.

Further support to the triple junction hypothesis was found in IV curves. Before irradiation, the pad leakage current presents a rapid increase at the multiplication-onset voltage, and then it continues to increase slowly, remaining almost constant. After irradiation, the general behaviour of the pad leakage current is the same. These results, which can be seen clearly in Fig. 10b, imply that the multiplication layer is, in fact, depleted and active at the same voltage even after irradiation. However, as observed in TCT voltage scans, this activation of the multiplication layer is not observed in TCT measurements. It might be that the defects caused to the bulk by radiation may be changing how the signal propagates inside the sensor, thus not allowing the measurements of the signal at voltages below full depletion. As regards the space charge change from the signinverted positive state (n-like) to the original negative state (p-type bulk), this could be explained based on the characteristic of the deep-level traps. If the occupation probability of the produced traps is highly dependent on the electric field, the increase in voltage may reduce the number of trapped holes. This would cause a flip in space charge sign, with the bulk going back to the original p-type behaviour.

\section{Conclusions}

LGADs with a multiplication layer dose of $1.8 \times 10^{13} \mathrm{~cm}^{-2}$, and $2.0 \times 10^{13} \mathrm{~cm}^{-2}$ were irradiated with $24-\mathrm{GeV} / \mathrm{c}$ protons. The devices were characterised before and after irradiation. The main focus of this study was to better understand the change in multiplication-onset voltage with irradiation, and the associated space charge changes in the samples irradiated up to $10^{14} \mathrm{n}_{\mathrm{eq}} / \mathrm{cm}^{2}$. TCT measurements show that after irradiation the multiplication-onset voltage increases, which is consistent with space charge sign inversion of the initially p-type bulk towards n-type. Furthermore, through TPA-TCT measurements and TCAD simulations, which allowed the analysis of the electric field evolution inside one of the studied LGADs, it was possible to infer that there is a triple junction effect occurring in the type inverted device. Interestingly, it was also discovered that with annealing the multiplication-onset voltage decreases, and the gain is recovered to $60-70 \%$ of the value before irradiation. As samples get annealed, they tend towards an unirradiated-like state. This implies that annealing is playing an important role for LGAD performance and might be exploitable for 
LGAD long term operation. Further irradiation campaigns and systematic annealing studies should be performed on similar samples to better comprehend these phenomena.

\section{Acknowledgment}

This work was developed in the framework of the CERN RD50 Collaboration, and has been partially supported by the Spanish Ministry of Economy and Competitiveness (grants FPA201571292-C2-1-P and FPA2015-69260-C3-3-R) and the European H2020 project AIDA-2020, GA no. 654168 .

\section{References}

[1] H.F.-W. Sadrozinski et al., Sensors for ultra-fast silicon detectors, Nucl. Instrum. Meth. A 765 (2014) $7-11$.

[2] G. Pellegrini et al., Technology developments and first measurements of Low Gain Avalanche Detectors (LGAD) for high energy physics applications, Nucl. Instrum. Meth. A 765 (2014) 12-16.

[3] G. Kramberger et al., Radiation effects in Low Gain Avalanche Detectors after hadron irradiations, JINST 10 (2015) P07006.

[4] E. Cavallaro et al., First measurements of segmented silicon tracking detectors with built-in multiplication layer, Nucl. Instrum. Meth. A 796 (2015) 136 - 140.

[5] G. Pellegrini et al., Recent technological developments on LGAD and iLGAD detectors for tracking and timing applications, Nucl. Instrum. Meth. A 831 (2016) 24 - 28.

[6] N. Cartiglia et al., Beam test results of a 16ps timing system based on ultra-fast silicon detectors, Nucl. Instrum. Meth. A 850 (2017) 83 - 88.

[7] C. Gallrapp et al., Study of gain homogeneity and radiation effects of Low Gain Avalanche Pad Detectors, Nucl. Instrum. Meth. A 875 (2017) 27 - 34.

[8] S. Otero Ugobono et al., Radiation Tolerance of Proton-Irradiated LGADs, IEEE Transactions on Nuclear Science (2018) 1-1.

[9] ATLAS Collaboration collaboration, C. Agapopoulou, A High-Granularity Timing Detector for the Phase-II upgrade of the ATLAS Detector System, Tech. Rep. ATL-LARG-PROC-2017-004, CERN, Geneva, Nov, 2017.

[10] The CMS and TOTEM Collaborations, CMS-TOTEM Precision Proton Spectrometer, Tech. Rep. CERN-LHCC-2014-021. TOTEM-TDR-003. CMS-TDR-13, Sep, 2014.

[11] CMS Collaboration, Technical Proposal for a MIP Timing Detector in the CMS Experiment Phase 2 Upgrade, Tech. Rep. CERN-LHCC-2017-027. LHCC-P-009, CERN, Geneva, Dec, 2017.

[12] V. Greco et al., Silicon Devices Optimised for Avalanche Multiplication, in proceedings of The 23rd International Workshop on Vertex Detectors, PoS(Vertex2014)031 (2015).

[13] V. A. K. Temple, Junction termination extension (JTE), A new technique for increasing avalanche breakdown voltage and controlling surface electric fields in $P-N$ junctions, in 1977 International Electron Devices Meeting, vol. 23, pp. 423-426, 1977, DOI.

[14] CERN PS IRRAD Proton Facility, https://ps-irrad.web.cern.ch/ . 
[15] I. Mateu et al., NIEL hardness factor determination for the new proton irradiation facility at CERN, presented at the 28th RD50 Workshop, Torino, Italy, 2016.

[16] V. Eremin et al., Development of transient current and charge techniques for the measurement of effective net concentration of ionized charges (Neff) in the space charge region of p-n junction detectors, Nucl. Instrum. Meth. A 372 (1996) 388-398.

[17] G. Kramberger et al., Investigation of Irradiated Silicon Detectors by Edge-TCT, IEEE Transactions on Nuclear Science 57 (Aug, 2010) 2294-2302.

[18] I. Vila et al., TPA-TCT: A novel Transient Current Technique based on the Two Photon Absorption (TPA) process, presented at the 25th RD50 Workshop, Geneva, Switzerland, 2014.

[19] M. Göppert-Mayer, Über elementarakte mit zwei quantensprüngen, Annalen der Physik 401 (1931) 273-294.

[20] H. Spieler, Semiconductor Detector Systems. Oxford University Press, Oxford, Great Britain, 2005.

[21] V. Eremin, E. Verbitskaya and Z. Li, The origin of double peak electric field distribution in heavily irradiated silicon detectors, Nucl. Instrum. Meth. A 476 (2002) 556 - 564.

[22] G. Kramberger et al., Effects of irradiation on LGAD devices with high excess current, presented at the 25th RD50 Workshop, Geneva, Switzerland, 2014.

[23] M. Fernández García, M. Moll and S. Otero Ugobono, The Transient Current Technique: laser characterization of silicon detectors, presented at DT Training Seminars, November 30th 2017, CERN, Geneva, Switzerland, https://indico.cern.ch/event/684193/. 